\title{
Yeast Water Relations: Physiological Changes Induced by Solute Stress in Saccharomyces cerevisiae and Saccharomyces rouxii
}

\author{
By MARGARET EDGLEY AND A. D. BROWN* \\ Department of Biology, University of Wollongong, Wollongong, NSW 2500, Australia
}

(Received 11 January 1983; revised 23 May 1983)

\begin{abstract}
A comparison was made of the xerotolerant yeast Saccharomyces rouxii and its non-tolerant counterpart $S$. cerevisiae when fully adapted to growth media of high and low water activity $\left(a_{\mathrm{w}}\right)$, and during the period of adaptation from a high $a_{\mathrm{w}}$ to a medium containing $\mathrm{NaCl}(10 \%, \mathrm{w} / \mathrm{v})$. Comparisons of the fully adapted yeasts were confined to levels of activity of phosphofructokinase and $s n$-glycerol-3-phosphate dehydrogenase. The results confirmed our previous findings obtained with different assay procedures that growth at diminished $a_{\mathrm{w}}$ dramatically increased the level of glycerol phosphate dehydrogenase in $S$. cerevisiae but had little or no effect in that respect on $S$. rouxii. Simultaneously phosphofructokinase activity was about doubled in $S$. cerevisiae and about halved in $S$. rouxii. Differences between the two yeasts were even more conspicuous during the period of response to salt stress. Saccharomyces rouxii apparently adapted fully to the salt broth within $6 \mathrm{~h}\left(\right.$ at $30^{\circ} \mathrm{C}$ ) inasmuch as viability was unaffected by the transfer and glycerol content reached its maximum by then. On the other hand, S. cerevisiae took about $140 \mathrm{~h}$ to adapt by a process that could be resolved into two stages. Stage 1 was marked by a catastrophic drop in apparent viability and widely different counts on three plating media. Stage 2 was identified by similar counts on all three plating media and a progressive increase in viability up to the level of the original inoculum. Glycerol phosphate dehydrogenase activity increased during this stage but glycerol accumulation began in both species immediately after transfer. Throughout the entire adaptation period, $S$. cerevisiae consumed glucose much more slowly than did $S$. rouxii. The complexity of the response of $S$. cerevisiae to salt stress has focused attention on this species as a reference for studying yeast water relations in general.
\end{abstract}

\section{INTRODUCTION}

The two yeasts Saccharomyces rouxii and $S$. cerevisiae differ considerably in their water relations: $S$. rouxii is highly xerotolerant whereas $S$. cerevisiae is relatively intolerant of diminished water activity $\left(a_{\mathrm{w}}\right)$. In spite of this, both species osmoregulate with glycerol, which also functions as a compatible solute under relatively extreme conditions of low $a_{\mathrm{w}}$. There are important physiological differences in the osmoregulatory mechanisms, however: $S$. rouxii responds to a solute stress by increasing the intracellular proportion of a constant amount of glycerol, whereas $S$. cerevisiae responds by retaining a constant intracellular proportion of an increased amount of glycerol which it synthesizes. It is reasonably certain that the major redirection of its carbohydrate metabolism to glycerol production in response to a solute stress is an important factor limiting the tolerance of $S$. cerevisiae to low $a_{\mathrm{w}}$ (Brown, 1978; Edgley \& Brown, 1978).

There are some fundamental biochemical differences that are associated with the different osmoregulatory mechanisms used by the two species. For example, Brown \& Edgley (1980) reported evidence of differences in the major metabolic pathways to glycerol, differences

Abbreviations: DHA, dihydroxyacetone; DHAP, dihydroxyacetone phosphate; MA, malt-extract agar; SHA, synthetic honey agar. 
between the species in the response to water stress of those pathways and differences in production of the enzyme $s n$-glycerol-3-phosphate dehydrogenase.

The present paper describes responses of this enzyme and phosphofructokinase to growth of each yeast at several levels of $a_{\mathrm{w}}$ and some of the changes that occur in each species during adaptation from a 'normal' growth medium $\left(a_{\mathrm{w}} 0.997\right)$ to one containing $\mathrm{NaCl}\left(10 \%, \mathrm{w} / \mathrm{v} ; a_{\mathrm{w}}\right.$ $0.936)$. The responses of the two yeasts during the transition period are very different in several respects and their water relations differ for complex reasons over and above mechanisms of controlling glycerol content.

\section{METHODS}

Organisms. Saccharomyces rouxii (strain YA, ATCC 38528) and Saccharomyces cerevisiae (strain Y41, ATCC 38531), described by Anand \& Brown (1968), were maintained at $4{ }^{\circ} \mathrm{C}$ on slopes of synthetic honey agar (SHA; containing 48\%, w/v, glucose; $a_{\mathrm{w}}$ 0.924; Anand \& Brown, 1968) and malt-extract agar (MA; Oxoid) respectively. Experimental cultures were grown in basal liquid medium ( $a_{\mathrm{w}}$ 0.997; Anand \& Brown, 1968), adjusted where necessary to the stated $a_{\mathrm{w}}$ by addition of $\mathrm{NaCl}$ or PEG (av. mol. wt 200), in conical flasks of nominal capacity 2.5 times the volume of the growth medium and incubated at $30{ }^{\circ} \mathrm{C}$ with rotary agitation (200 r.p.m.). PEG was added as an aqueous solution (50\%) that had previously been deionized on a mixed-bed ionexchange resin.

For experiments with organisms fully adapted to a specified $a_{\mathrm{w}}$ (steady-state experiments) an inoculation schedule as described by Edgley \& Brown (1978) was used. The inoculation and transfer procedures for experiments in which adaptation of a yeast from basal medium to the same medium containing $\mathrm{NaCl}(10 \%$, w/v) ('salt broth', $a_{\mathrm{w}}$ 0.936) was monitored (transition experiments) were as follows. Basal medium (100 ml) was inoculated by loop from a 1-2 d slope and grown overnight to stationary phase. A new batch of basal medium (400 $\mathrm{ml})$ was inoculated to a level of $10 \%(\mathrm{v} / \mathrm{v})$ with the first culture and grown well into the exponential growth phase (about $4.5 \mathrm{~h}$ and $2.5 \mathrm{~h}$ respectively for $S$. rouxii and $S$. cerevisiae). The viable count at this point was normally $6-8 \times 10^{6}$ $\mathrm{ml}^{-1}$. The culture was chilled in ice and centrifuged briefly at $13200 \mathrm{~g}$ at $0{ }^{\circ} \mathrm{C}$. The pellet was resuspended in salt broth $(400 \mathrm{ml})$ at $30^{\circ} \mathrm{C}$, and incubated normally. Aseptic precautions were used throughout the transfer.

Viable counts were made conventionally by the drop plate technique. Some comparisons were made with pour plates. Serial dilutions were made in quarter-strength Ringer's solution for counts of basal-medium cultures and in $\mathrm{NaCl}(10 \%, \mathrm{w} / \mathrm{v})$ for salt broth cultures. Suspensions were plated on to MA, SHA and salt-agar [basal medium with $\mathrm{NaCl}(10 \%, \mathrm{w} / \mathrm{v})$ and agar $(1.5 \%, \mathrm{w} / \mathrm{v})]$. Colonies on the three media were counted after, respectively, 3,5 and $18 \mathrm{~d}$ incubation at $30^{\circ} \mathrm{C}$. Salt-agar plates were incubated in plastic bags to restrict evaporation.

Enzyme assays. Cultures (approximately $400 \mathrm{ml}$ ) in the mid-exponential growth phase were chilled thoroughly in ice, centrifuged briefly at $13200 \mathrm{~g}$ at $0{ }^{\circ} \mathrm{C}$ then washed twice by brief centrifugation at $0{ }^{\circ} \mathrm{C}$ with either of the following buffer solutions: (i) sodium phosphate buffer $(0.067 \mathrm{M}, \mathrm{pH} \mathrm{7.0)}$ for subsequent assay of phosphofructokinase; (ii) triethanolamine/ $\mathrm{HCl}(10 \mathrm{mM}, \mathrm{pH} 7 \cdot 0)$ containing EDTA ( $1.0 \mathrm{mM})$ and dithiothreitol ( $1.0 \mathrm{mM})$ for subsequent assay of $s n$-glycerol-3-phosphate dehydrogenase. The washed yeast was disrupted with a modified Hughes press (Anand, 1969). The broken cells were dispersed in cold wash buffer $(0.8-1.0 \mathrm{ml})$ and centrifuged at $39100 \mathrm{~g}$ for $30 \mathrm{~min}$ at $0^{\circ} \mathrm{C}$. The extracts were kept at $0^{\circ} \mathrm{C}$ and assayed on the day of preparation. If necessary, extracts were diluted with wash buffer.

$s n$-Glycerol-3-phosphate dehydrogenase was assayed according to Gancedo et al. (1968). The method involved assessing the rate of reduction of dihydroxyacetone phosphate (DHAP) by NADH. Specific activity of glycerol-3phosphate dehydrogenase from $S$. cerevisiae increased with protein concentration as does the corresponding enzyme purified from rabbit muscle (Batke et al., 1980). The relation between protein concentration in crude extracts and specific activity of the $S$. cerevisiae enzyme(s) fitted the Michaelis-Menten equation and we were therefore able to use a double reciprocal plot to determine specific activity at infinite protein concentration. Specific activity of glycerol-3-phosphate dehydrogenase from $S$. cerevisiae only is thus expressed throughout as $\mathrm{SA}_{\max }$, that is the specific activity determined by extrapolation to infinite protein concentration. This was not done for the results reported by Brown \& Edgley (1980), nor, in the present work, with assays of DHAP reduction by NADPH.

Phosphofructokinase was assayed according to Sols \& Salas (1970) using a coupled enzyme system. The supplementary enzymes used in this assay (aldolase, triosephosphate isomerase and glycerol-3-phosphate dehydrogenase) were obtained commercially as suspensions in $\left(\mathrm{NH}_{4}\right)_{2} \mathrm{SO}_{4}(3.2 \mathrm{M})$ and, where indicated, were dialysed

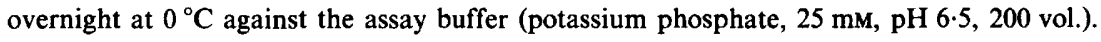

Analytical methods. Protein was estimated by the biuret method (Layne, 1957) using bovine serum albumin standards.

Polyols (total) were estimated by periodate oxidation (Hanahan \& Olley, 1958). Glycerol was estimated enzymically (Eggstein \& Kuhlmann, 1974) but with NADH at a concentration of $0.1 \mathrm{mM}$ in the assay. The amount 
of arabitol in $S$. rouxii extracts was calculated from the difference between total polyol and glycerol content, since these were the only polyhydric alcohols detectable in the extracts by chromatography. Arabitol was not detected in $S$. cerevisiae extracts.

Glucose was estimated enzymically with a commercial glucose oxidase kit (GOD-Perid, Boehringer) used as follows. The glucose oxidase reagent was dissolved in water to a concentration of $5 \%(\mathrm{w} / \mathrm{v})$. This solution $(0 \cdot 2 \mathrm{ml})$ was mixed with the sugar solution $(0.2 \mathrm{ml})$ and incubated with shaking for $30 \mathrm{~min}$ at $30^{\circ} \mathrm{C}$. Acetic acid $(3.6 \mathrm{ml})$ was added, and the $A_{650}$ was measured. (The wavelength can be changed if the readings are too high.) An $A_{650}$ of about 0.5 is given by $0.5 \mathrm{~mm}$ glucose. This modified use of the commercial reagent is based on the method of Davies \& Wayman (1973). Trehalose was provisionally identified by descending paper chromatography in butan-1-ol/acetic acid/water $(6: 1: 2$, by vol.) and detection with silver nitrate.

Preparation of extracts for analysis. Samples $(25 \mathrm{ml})$ of culture were chilled in ice and centrifuged at $12100 \mathrm{~g}$ for $10 \mathrm{~min}$. The supernatant solution was collected and immediately frozen. The pellets were washed twice at $0{ }^{\circ} \mathrm{C}$ with water (4.0 ml; samples from basal medium) or $10 \% \mathrm{NaCl}(4.0 \mathrm{ml}$; samples from salt broth) by centrifuging for $5 \mathrm{~min}$ at $12100 \mathrm{~g}$. The washings were pooled and frozen. The pellet was freeze-dried and extracted first by mixing with absolute ethanol $(10 \mathrm{ml})$ and allowing to stand overnight at $0{ }^{\circ} \mathrm{C}$. Water $\left(15 \mathrm{ml}, 0^{\circ} \mathrm{C}\right)$ was then added to this slurry, and the aqueous-ethanolic slurry was maintained at $0^{\circ} \mathrm{C}$ for $45 \mathrm{~min}$ after which it was centrifuged and washed twice in the centrifuge with water $(10 \mathrm{ml})$. The extract and washings were combined and concentrated by rotary evaporation under vacuum.

Intracellular polyol content was calculated from the polyol content of cell extracts plus that lost in washing. Polyol leakage during washing was estimated by subtracting from the amount in the washings the amount contributed by the growth medium retained as interstitial fluid after the first centrifugation. The volume of interstitial fluid was calculated from the amount of glucose in the washings and the concentration of glucose in the medium at the time of harvesting, on the assumption that all glucose in the washings had an extracellular origin.

Dry mass was determined on separate $25 \mathrm{ml}$ samples of culture by centrifuging and washing with water as described above and drying at $110^{\circ} \mathrm{C}$ for $24 \mathrm{~h}$.

\section{RESULTS}

\section{Enzyme activity in the fully adapted yeast}

Table 1 shows glycerol-3-phosphate dehydrogenase activity or, more precisely, specific rates of reduction of DHAP by unfractionated extracts of each yeast, with NADH and NADPH separately as reductants. The salient features of this table are: (i) in $S$. cerevisiae the enzyme activity was about 100 times greater with NADH than with NADPH; (ii) activity with NADH was increased by up to 30 -fold, and with NADPH by up to 20 -fold, when $S$. cerevisiae was grown in media containing $\mathrm{NaCl}$, the greatest increase occurring with $10 \%(\mathrm{w} / \mathrm{v}) \mathrm{NaCl}$; (iii) the

\section{Table 1. Effects of solute concentration or $a_{w}$ of the growth medium on DHAP-reducing activity of crude extracts of Saccharomyces cerevisiae and $S$. rouxii}

Activities are expressed as $\mathrm{SA}_{\max }$ (see Methods) for reduction of DHAP with NADH by $S$. cerevisiae extracts and conventionally as specific activity in all other cases. The units are nmol $\mathrm{min}^{-1}(\mathrm{mg}$ protein $)^{-1}$. The numbers in parentheses denote the number of preparations assayed. Values are given as mean \pm S.E.M. for four or more preparations and otherwise as the mean and range.

\begin{tabular}{|c|c|c|c|c|}
\hline \multirow[b]{3}{*}{ Growth medium } & \multicolumn{4}{|c|}{ Enzyme activity } \\
\hline & \multicolumn{2}{|c|}{ S. cerevisiae } & \multicolumn{2}{|c|}{ S. rouxii } \\
\hline & NADH & NADPH & NADH & NADPH \\
\hline $\begin{array}{l}\text { Basal } \\
\qquad\left(a_{w} 0.997\right)\end{array}$ & $154 \underset{(6)}{ \pm} 28$ & $2 \cdot 1 \underset{(6)}{ \pm} 0 \cdot 2$ & $7 \cdot 0 \underset{(4)}{ \pm} 1 \cdot 0$ & $4 \cdot 5 \underset{(4)}{ \pm} 0 \cdot 8$ \\
\hline $\begin{array}{l}\text { Salt broth } \\
\left(5 \% \mathrm{NaCl}, a_{\mathrm{w}} 0.968\right)\end{array}$ & $1182 \pm \frac{ \pm}{(2)} 62$ & $\begin{array}{l}8 \cdot 1 \\
(1)\end{array}$ & $\begin{array}{l}6 \cdot 5 \\
(1)\end{array}$ & $\begin{array}{l}2 \cdot 4 \\
(1)\end{array}$ \\
\hline $\begin{array}{l}\text { Salt broth } \\
\left(10 \% \mathrm{NaCl}, a_{\mathrm{w}} 0.936\right)\end{array}$ & $4598 \underset{(2)}{ \pm} 214$ & $\begin{array}{l}46 \cdot 5 \\
(1)\end{array}$ & $\begin{array}{l}11 \cdot 2 \\
(1)\end{array}$ & $\begin{array}{l}2 \cdot 0 \\
(1)\end{array}$ \\
\hline $\begin{array}{l}\text { Basal }+ \text { PEG } \\
\left(a_{w} 0.970\right)\end{array}$ & $990 \pm 74$ & - & $\begin{array}{l}10 \cdot 3 \\
(1)\end{array}$ & $\begin{array}{l}4 \cdot 5 \\
(1)\end{array}$ \\
\hline $\begin{array}{l}\text { Basal }+ \text { PEG } \\
\left(a_{\mathrm{w}} 0.940\right)\end{array}$ & $1340 \pm 24$ & - & $\begin{array}{l}8 \cdot 8 \\
(1)\end{array}$ & $\begin{array}{l}5 \cdot 2 \\
(1)\end{array}$ \\
\hline
\end{tabular}


Table 2. Effects of growth in the presence of salt on phosphofructokinase activity in crude extracts of Saccharomyces cerevisiae and $S$. rouxii

Units of specific activity are $\mathrm{nmol} \mathrm{min}^{-1}(\mathrm{mg} \text { protein })^{-1}$. Values for basal medium preparations are given as the mean and S.E.M. for three preparations. Values for salt broth are the mean and range for two preparations. Inhibition by $1 \mathrm{mM}-\mathrm{ATP}$ was calculated with reference to rates obtained with $0.1 \mathrm{~mm}-$ ATP.

\begin{tabular}{|c|c|c|c|c|c|c|}
\hline \multirow[b]{2}{*}{ Growth medium } & \multicolumn{2}{|c|}{ Assay conditions } & \multicolumn{2}{|c|}{ Specific activity } & \multicolumn{2}{|c|}{ Inhibition by $1 \mathrm{~mm}-\mathrm{ATP}(\%)$} \\
\hline & $\begin{array}{c}\mathrm{NH}_{4}^{+} \\
(16 \mathrm{mM})\end{array}$ & $\begin{array}{l}\text { [ATP] } \\
(\mathrm{mM})\end{array}$ & S. cerevisiae & $S$. rouxii & S. cerevisiae & $S$. rouxii \\
\hline $\begin{array}{l}\text { Basal } \\
\qquad\left(a_{\mathrm{w}} 0.997\right)\end{array}$ & $\begin{array}{l}+ \\
+ \\
- \\
-\end{array}$ & $\begin{array}{l}0.1 \\
1.0 \\
0.1 \\
1.0\end{array}$ & $\begin{array}{r}168 \pm 20 \\
157 \pm 37 \\
138 \pm 6 \\
14 \pm 5\end{array}$ & $\begin{array}{r}145 \pm 11 \\
51 \pm 8 \\
10 \pm 1 \\
2 \pm 1\end{array}$ & $\frac{7}{9}$ & $\frac{\overline{65}}{\overline{80}}$ \\
\hline $\begin{array}{l}\text { Salt broth } \\
\left(10 \% \mathrm{NaCl}, a_{\mathrm{w}} 0.936\right)\end{array}$ & $\begin{array}{l}+ \\
+\end{array}$ & $\begin{array}{l}0 \cdot 1 \\
1 \cdot 0\end{array}$ & $\begin{array}{l}300 \pm 40 \\
174 \pm 23\end{array}$ & $\begin{array}{l}92 \pm 5 \\
36 \pm 4\end{array}$ & $\overline{42}$ & $\overline{61}$ \\
\hline
\end{tabular}

activity of $S$. cerevisiae extract was 20-400 times that of $S$. rouxii, depending on growth conditions. In $S$. rouxii, enzyme activity with NADH was $1.5-5.5$ times as great as with NADPH and neither was appreciably affected by $a_{\mathrm{w}}$ or salinity.

Table 2 lists phosphofructokinase activity in extracts of the two yeasts after growth in basal medium and salt broth. Features of this table include the degree of inhibition effected by the substrate, ATP, and the influence on that inhibition of lowering the $\mathrm{NH}_{4}^{+}$concentration by dialysing the supplementary enzymes. Phosphofructokinase from many sources is subject to substrate inhibition by ATP (Hofman, 1976) and, for that reason, the enzyme is sometimes assayed with GTP. Inhibition by ATP is reversed, at least for baker's yeast phosphofructokinase, by $\mathrm{NH}_{4}^{+}$(Atzpodian \& Bode, 1970).

There was apparently a different type of phosphofructokinase in each species, that in $S$. rouxii being much more susceptible to ATP inhibition than the enzyme(s) from $S$. cerevisiae. This difference was exaggerated when dialysed auxiliary enzymes were used and, without the protection of $\mathrm{NH}_{4}^{+}$, the $S$. cerevisiae preparation was also substantially inhibited by $1.0 \mathrm{mM}-$ ATP. The effect was reversed by restoring $\mathrm{NH}_{4}^{+}$(added as $\mathrm{NH}_{4} \mathrm{Cl}$ ) to the assay mixture. When the yeasts were grown in salt broth, phosphofructokinase activity was about halved in $S$. rouxii and about doubled in $S$. cerevisiae. The additional activity so produced, however, was sensitive to $1.0 \mathrm{mM}$-ATP (assayed with undialysed supplementary enzymes), implying two types of phosphofructokinase and a greater potential for regulation of glycolysis when $S$. cerevisiae is grown in $10 \% \mathrm{NaCl}$.

\section{Changes during adaptation to salt broth}

Viability changes. When $S$. rouxii was transferred to salt broth (containing $10 \% \mathrm{NaCl}$ ), viability, as assessed by plate counts on three agar media, changed very little and there was reasonably close agreement among the three media (Fig. 1a). The approximately twofold increase in the population during the period of observation was as expected since the inoculating culture was harvested somewhat more than half-way through its exponential growth phase.

The response of the population of $S$. cerevisiae to transfer to salt broth was totally different (Fig. $1 b$ ). We have designated the first $45 \mathrm{~h}$, with widely different plate counts, as Stage 1, and the period after $45 \mathrm{~h}$ as Stage 2 of the adaptation process. Similar results were obtained when the inoculum was grown from a single colony instead of a slope culture (see Methods).

Polyols and trehalose. Accumulation of intracellular glycerol by each species began immediately after transfer to salt broth, or at least within the sampling time of about $3 \mathrm{~min}$ (Table 3; Fig. 2). In $S$. rouxii (Table $3 a$ ) there was some synthesis of both glycerol and arabitol during the first $5 \mathrm{~h}$ or so after transfer. The change in the quotient intracellular/extracellular glycerol refiected initially the delay in accumulation of glycerol outside the cell (over the first 

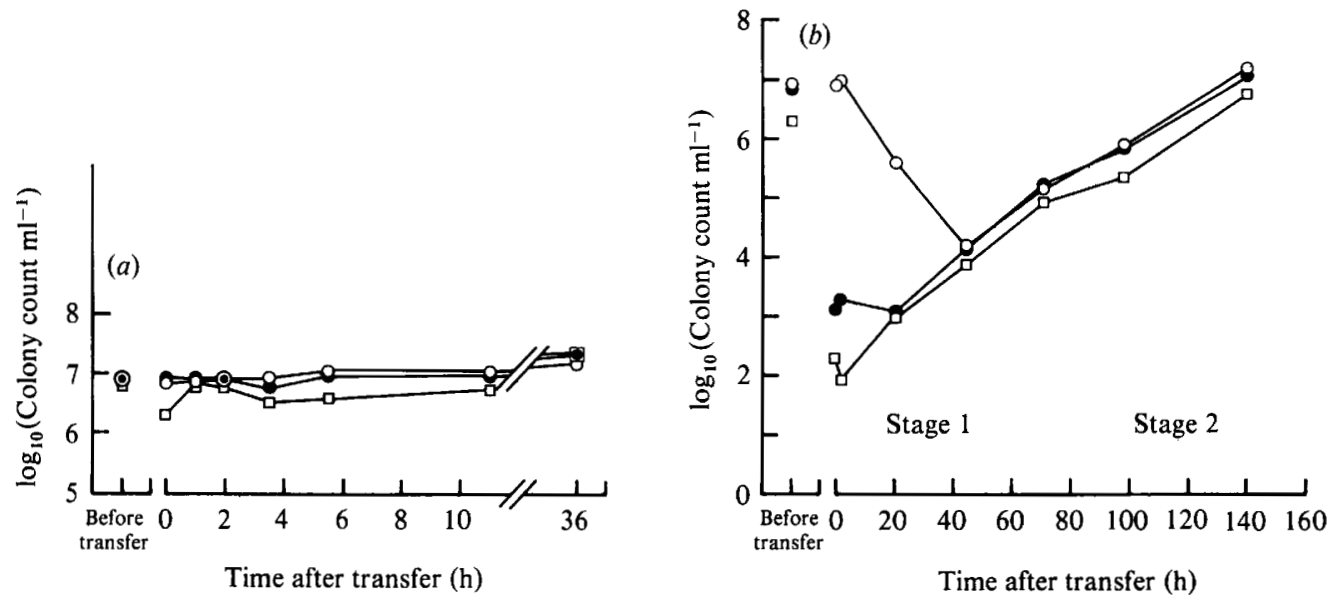

Fig. 1. Viable counts, on three agar media, of Saccharomyces rouxii (a) and S. cerevisiae (b) after transfer from basal yeast medium to salt broth (containing $10 \%, \mathrm{w} / \mathrm{v}, \mathrm{NaCl}$ ). The 'before transfer' counts were those of the inoculating culture before harvesting (see text). $O$, Count on malt extract agar; $P$, count on synthetic honey agar; $\square$, count on salt agar.

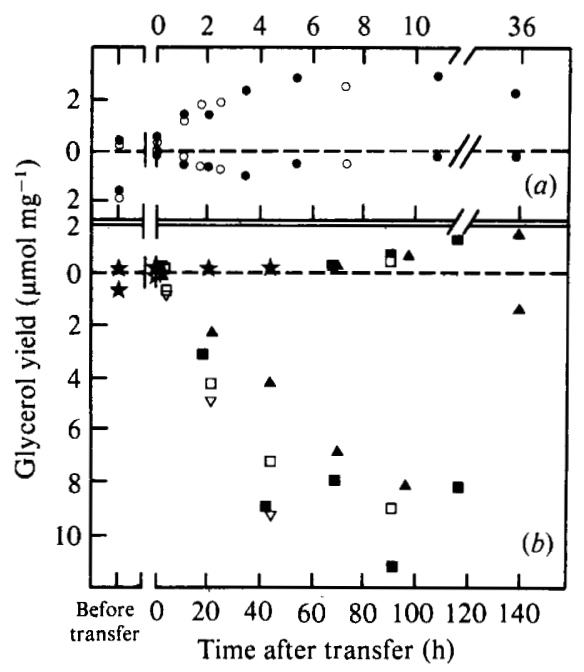

Fig. 2. The partitioning of glycerol in Saccharomyces rouxii (a) and S. cerevisiae (b) after transfer from basal yeast medium to salt broth (containing $10 \%, \mathrm{w} / \mathrm{v}, \mathrm{NaCl}$ ). Points above the zero line (dashed) denote intracellular glycerol content and, below the line, yield of extracellular glycerol expressed, in the same units, as a function of the mass of dry yeast. The 'before transfer' values were those of the inoculating culture before harvesting. Note the different time scales in parts $(a)$ and $(b)$. This figure shows graphically for several experiments (denoted by the various symbols) results similar to those listed for single experiments in Table $3(a$ and $b)$. The stars denote the effective coincidence of four points.

$3.5 \mathrm{~h})$ and later the consumption or uptake of extracellular glycerol. The corresponding ratio for arabitol decreased over the period $1-11 \mathrm{~h}$, reflecting a relatively stable internal arabitol content and a progressive increase in the amount of extracellular arabitol. Ultimately external arabitol was also consumed.

Synthesis of glycerol by $S$. cerevisiae in response to the stress was much slower than in $S$. rouxii even though it, too, began immediately after the transfer (Table $3 b$ ). Total synthesis continued 


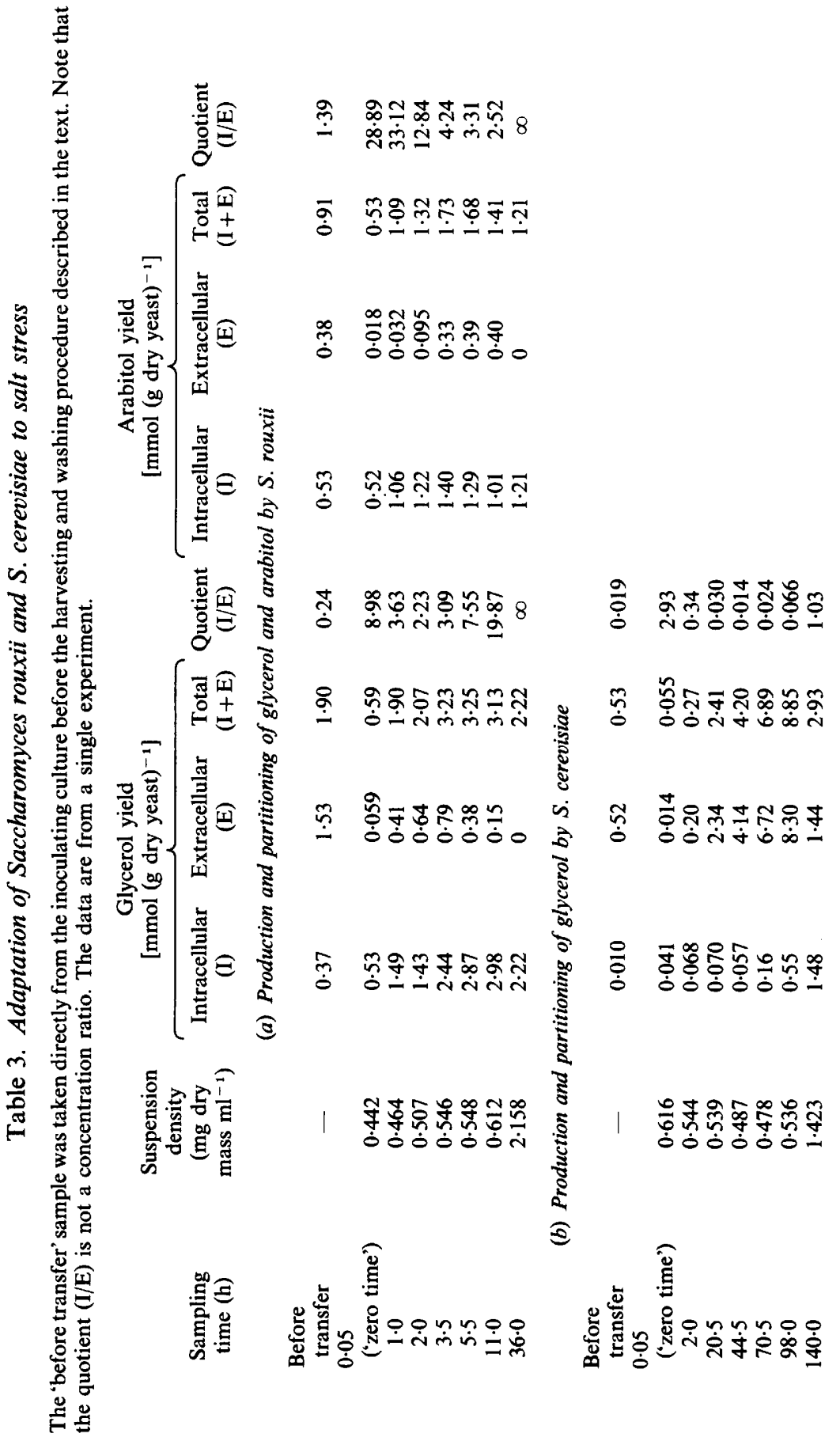




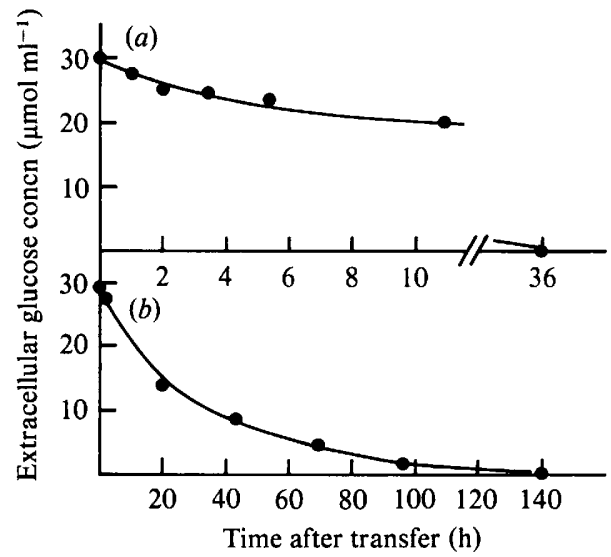

Fig. 3. Consumption of glucose by Saccharomyces rouxii $(a)$ and $S$. cerevisiae $(b)$ after transfer from basal yeast medium to salt broth. The results in $(a)$ are from the same experiment as shown in Table 3(a) and Fig. 1(a) and, in $(b)$ Table $3(b)$ and Fig. $1(b)$. Note the different time scales in parts $(a)$ and $(b)$.

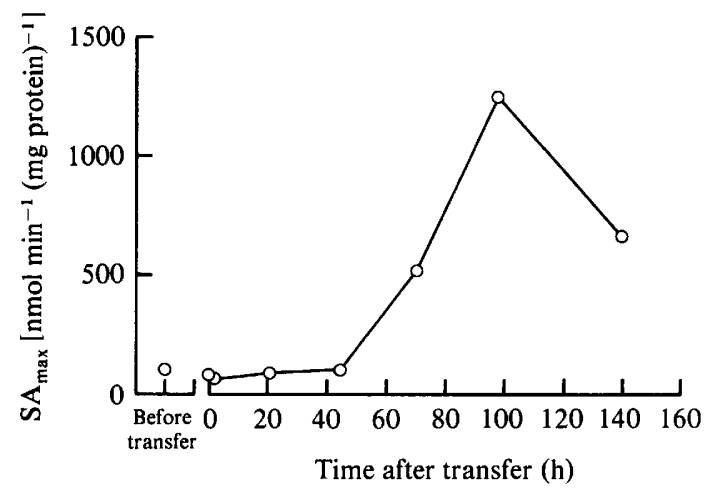

Fig. 4. Changes in the specific activity ( $\mathrm{SA}_{\max }$, see Methods) of glycerol-3-phosphate dehydrogenase after transfer of Saccharomyces cerevisiae from basal yeast medium to salt broth $(10 \%, \mathrm{w} / \mathrm{v}, \mathrm{NaCl})$. These results are from the same experiment as shown in Table $3(b)$ and Fig. $1(b)$.

for about $98 \mathrm{~h}$, however, and led to an amount nearly three times as much as produced by the xerotolerant species. Internal glycerol remained lower than in $S$. rouxii; external glycerol was much higher after the first $2 \mathrm{~h}$ and correspondingly the intracellular/extracellular quotient was low, being less than unity for all except the initial ('zero time') sample, when there was scarcely any external glycerol, and the $140 \mathrm{~h}$ sample, when external glycerol was being consumed. The quotient for $S$. rouxii was always substantially greater than unity. For $S$. cerevisiae, the quotient decreased during Stage 1 and increased during Stage 2 of the adaptation. A substance provisionally identified as trehalose was detectable by paper chromatography of extracts from $20 \mathrm{~h}$ onwards. This has previously been reported for fully adapted cultures of $S$. cerevisiae at low $a_{\mathrm{w}}$ (Brown, 1978). None has been detected in $S$. rouxii.

Glucose consumption. During the period of adaptation to salt broth $S$. rouxii consumed $30 \%$ of its extracellular glucose by $11 \mathrm{~h}$ and all of it by $36 \mathrm{~h}$ (Fig. $3 a$ ). For $S$. cerevisiae extracellular glucose concentration was about $9 \mu \mathrm{mol} \mathrm{ml}^{-1}$ (more than $30 \%$ of the initial concentration) at the end of Stage $1\left(45 \mathrm{~h}\right.$ ), less than $1 \mu \mathrm{mol} \mathrm{ml^{-1 }}$ at $100 \mathrm{~h}$ and totally consumed by $140 \mathrm{~h}$ (Fig. $3 b$ ).

Glycerol-3-phosphate dehydrogenase. There was no change in glycerol-3-phosphate dehydrogenase activity of $S$. cerevisiae during Stage 1 ; activity of the enzyme increased with Stage 2 and reached an apparent maximum at about $100 \mathrm{~h}$ (Fig. 4). These results are from the experiment 
represented in Table $3(b)$. Similar results were obtained from other experiments summarized in Fig. $2(b)$. There was no change in activity of $S$. rouxii extracts during the adaptation period.

\section{DISCUSSION}

The steady-state situation. The specific activities of glycerol-3-phosphate dehydrogenase shown in Table 1 are much higher for $S$. cerevisiae and slightly higher for $S$. rouxii than reported by Brown \& Edgley (1980). The main reasons for this are the use of a different extraction buffer [Brown \& Edgley (1980) used phosphate buffer (Sørensen, $\mathrm{Na}^{+}+\mathrm{K}^{+}, 0.067 \mathrm{M}, \mathrm{pH} 7.0$ ) in preparation of yeast extracts for enzyme assay], and the use of $\mathbf{S A}_{\max }$ in reporting the present results (see Methods). Glycerol-3-phosphate dehydrogenase from various sources has regulatory characteristics (e.g. Black, 1966; Tajima \& Yoshizumi, 1973; Bewley \& Miller, 1979; Nader et al., 1979; Storey \& Storey, 1982). The regulatory mechanism seems to include, at least for the enzyme from rabbit skeletal muscle, a capacity for aggregation-disaggregation between a monomeric and dimeric form of the enzyme as a function of both enzyme and substrate concentration. Both forms are active but the dimer is more so. The dimer can also apparently aggregate with aldolase to give a yet slightly more active form (Batke $e t$ al., 1980). The enzyme from $S$. cerevisiae is capable of somewhat similar behaviour, at least to the extent of apparently changing its aggregation state as a function of protein concentration. We have no evidence for or against its complexing specifically with another enzyme. Saccharomyces rouxii extracts, however, did not respond in this way to protein concentration with either NADH or NADPH as reductant and, at least by this criterion, the glycerol phosphate dehydrogenase from that species is a distinctly different enzyme(s) from that in $S$. cerevisiae.

Nevertheless, the general effects of growth at diminished $a_{\mathrm{w}}$ were essentially as described by Brown \& Edgley (1980) inasmuch as the specific activity of the glycerol phosphate dehydrogenase was greatly increased in $S$. cerevisiae (about 30 -fold in $10 \% \mathrm{NaCl}$ ) but was scarcely affected in $S$. rouxii. The stimulatory effect of PEG at the same $a_{\mathrm{w}}$ was somewhat less but still substantial. A difference between the two solutes is consistent with various other responses of yeast (Brown, 1978). The major effect of the different assay procedure on the results for $S$. rouxii was to reverse the apparent relative importance of NADPH and NADH in the reduction of DHAP. In the present series of assays activity was higher with NADH than with NADPH, whereas the reverse was true in Brown \& Edgley's (1980) results. In view of the difficulties inherent in comparing physiological activities of different reactions, it is reasonable to conclude that DHAP reduction in $S$. rouxii occurs with about equal facility with either NADH or NADPH as reductant and, under all experimental conditions, at a much lower rate than the NADH-specific reduction of DHAP in $S$. cerevisiae. This, together with the substantial rate of reduction of DHA and glyceraldehyde by NADPH and the relatively high levels of activity of the pentose phosphate cycle enzymes in $S$. rouxii (Brown \& Edgley, 1980), is consistent with a greater contribution by that cycle to carbohydrate metabolism in $S$. rouxii than in $S$. cerevisiae.

The specific activities of phosphofructokinase in Table 2 also imply that glycolysis is considerably less important for $S$. rouxii than for $S$. cerevisiae and, moreover, is more susceptible to control by ATP inhibition in the xerotolerant species. Furthermore, production of this enzyme, like that of glycerol phosphate dehydrogenase, was enhanced by growth in $10 \% \mathrm{NaCl}$ only in S. cerevisiae. The additional kinase so produced, however, had different substrate (ATP)inhibition characteristics from the dominant enzyme in the yeast grown on basal medium.

Thus important differences between the two species include not only the relative contributions of the possible metabolic pathways to glycerol and the major response of glycerol synthesis to $a_{\mathrm{w}}$ in only one of these species, but also effects of water activity or salinity on the production by $S$. cerevisiae of at least two enzymes involved in glycerol synthesis.

The transition. The transitional behaviour of $S$. cerevisiae compared with that of $S$. rouxii is a splendid example of what not to do if you want to be xerotolerant. Saccharomyces cerevisiae clearly 'recognized' a need for intracellular glycerol but its response to that need was much more complex and less effective than the simpler tactic used by $S$. rouxii. 
Nowhere is the difference between the two species more conspicuous than in the viability changes that occurred during the period of adaptation to salt broth. Whereas the population of $S$. rouxii was unruffled by the transfer, that of $S$. cerevisiae experienced a major trauma.

The information contained in Fig. $1(a$ and $b)$ is quite complex. For a start, there are several time factors implicit in the two figures. These are: (i) the time shown on the abscissa, which is the period spent by the yeast in the salt broth up to the moment of sampling; (ii) a period of minutes during the serial dilutions in $10 \% \mathrm{NaCl}$; (iii) the period of incubation of the plates which ranged from $3 \mathrm{~d}$ for MA to $18 \mathrm{~d}$ for salt agar. Thus the results reflect not only the state of adaptation of the population to the salt broth at the time of sampling but also, where appropriate, a continuation of the adaptation process in the discrete colony-forming units once plated on to the agar.

Plating from salt broth on to MA subjected the yeasts to a dilution stress (an osmotic shock) whereas plating on to salt agar or SHA did not. It is noteworthy in this context that, for the first $2 \mathrm{~h}$ after transfer, the count of $S$. cerevisiae on MA did not drop whereas on salt agar it did so catastrophically (by $>10^{4}$ ). Conversely, as the adaptation of $S$. cerevisiae progressed during Stage 1 the MA count dropped while that on salt agar (and, with some minor variations, on SHA) increased. This raises two questions, namely: (a) was the drop in the MA count during Stage 1 merely the result of a dilution stress as the adaptation progressed or was it a genuine expression of diminished total viability? (b) did the small residue of cells viable on salt agar and SHA represent a 'rump' of the population that was viable in salt broth and whose subsequent multiplication was actually the adaptation process or did it reflect merely the heterogeneity of a population that was severely stressed but which, in toto, adapted physiologically?

We do not yet know the answer to question (a), but the decrease in cell mass during the first $70 \mathrm{~h}$ or so of the adaptation of $S$. cerevisiae (Table $3 b$ ) indicates some loss of viability during this period. The declining count on MA, however, was accompanied by increasing counts on the other two media. The correlation between these two opposing trends must reflect in some way the physiological states of the population during Stage 1. Thus Stage 1 seems to be a series of 'points of no return' beyond which part of the population sacrifices its recovery potential (count on MA) in the interests of increasing its apparent viability in the salt medium. Once into Stage 2, this trade-off is no longer necessary.

There is some evidence relevant to question (b). First, the apparent exponential growth rate of the population during late Stage 1 and early Stage 2 was about $0 \cdot 14$ generations $\mathrm{h}^{-1}$, which is low enough to be consistent with either multiplication or physiological adaptation. Second, K. F. McKenzie \& A. D. Brown (unpublished) have shown that, in the course of a normal growth cycle in basal medium $S$. cerevisiae, but not $S$. rouxii, passes through a period in which counts on SHA and salt agar are at least $10^{3}$ below counts on MA. The period coincides with bud formation and recovery is rapid when the buds separate. This observation seems to embody part of an explanation of the events depicted in Fig. $1(b)$ and, for the present at least, we regard the apparent population changes of Stage 1 as a reflection of a general physiological adaptation of the whole population rather than simply the multiplication of a small viable residue.

Another factor that is relevant to this question is the glycerol content of the yeast if glycerol is produced only by those cells that are viable by any of the criteria that might be invoked from Fig. $1(b)$. When this is done, taking the average mass of a wet yeast cell to be about $2 \times 10^{-10} \mathrm{~g}$ and the water content to be about $60 \%$, then apparent intracellular glycerol concentrations (from Table $3 \mathrm{~b}$ ) for viable cells are as follows : $2 \mathrm{~h}$, MA count (as index of viability), 0.03 molal; salt agar count, $3 \times 10^{3}$ molal; $44.5 \mathrm{~h}$, MA count, 23 molal. Except when the $2 \mathrm{~h}$ count on MA was used as the number of viable cells, these values are patently impossible. We must therefore conclude that glycerol is produced essentially by the whole population of $S$. cerevisiae and not solely by that portion that is viable by any of the criteria employed.

Although the anomalies in viability that are characteristic of the early period of the transition were overcome by the end of Stage 1, Stage 2 was not simply a period of multiplication by the fully adapted organisms since the yeast produced both additional glycerol and glycerol phosphate dehydrogenase during this period (Table $3 b$; Fig. 4). Although we have not followed changes in phosphofructokinase activity during the transition, it is reasonable to assume that the 
additional enzyme indicated in Table 2 is also produced during Stage 2 . Table 2 implies that the extra phosphofructokinase produced by the fully adapted yeast is an isoenzyme of that produced in basal medium, since it was affected differently by ATP. It is therefore pertinent to ask whether the additional glycerol phosphate dehydrogenase produced in response to salt stress was more of the same or also an isoenzyme of that produced in basal medium. We cannot yet answer this question but isoenzymes of glycerol phosphate dehydrogenase are common in other organisms, especially when activity is increased in some tissues at certain periods of development (e.g. Bewley \& Miller, 1979).

Although we do not yet have direct evidence in a number of relevant areas, it is obvious that, during Stage 1, S. cerevisiae is capable of glycerol production, and hence energy metabolism, but may well be incapable of protein synthesis. We have not yet determined whether the additional glycerol phosphate dehydrogenase activity that appeared in Stage 2 was the result of protein synthesis or of a process such as the activation of a zymogen. For the present we assume, as an hypothesis, that it was de novo synthesis. Glycerol production continued during Stage 2 but its content for much of the time was less than that appropriate for the prevailing $a_{\mathrm{w}}$. We must assume, therefore, that intracellular physical chemistry was more inhibitory than in the fully adapted yeast but, of course, less inhibitory than in the early part of Stage 1. In spite of this, not only is energy metabolism possible but so is enhancement of enzyme activity.

Thus the overall response of $S$. cerevisiae is determined by at least two environmental signals, an immediate one redirecting carbohydrate metabolism to glycerol production and a later one leading to an increase in enzyme activity. We have already suggested that the former might be $\mathrm{K}^{+}$depletion and the latter increased intracellular glycerol concentration (Brown \& Edgley, 1980).

In comparison with the complexity of the attempt by $S$. cerevisiae to adapt to $10 \% \mathrm{NaCl}$, there is little to be said about the effortless transition of $S$. rouxii. Viability was not affected by the transfer. We have previously reported (Brown, 1978; Edgley, 1980) that fully adapted $S$. rouxii does not significantly vary the amount of polyol it synthesizes in relation to the $a_{\mathrm{w}}$ of its growth medium and that it varies its glycerol content merely by controlling the proportion it retains of an approximately constant amount synthesized. The present results (Table $3 a$ ) show that it did, in fact, synthesize both arabitol and glycerol during the transition although their combined amount was less than half the glycerol production of $S$. cerevisiae. In spite of this, $S$. rouxii produced its glycerol much faster than $S$. cerevisiae during the transition. Since the culture conditions used in the present work were quite different from those used previously for the fully adapted yeast in steady state, and because the extracellular glycerol load of the inoculating culture had been depleted, differences between the two sets of conditions in the several variables studied are to be expected. The constant factor is that, although $S$. rouxii did synthesize some glycerol during adaptation to salt stress, it made use of enhanced retention as a major contribution to raising its glycerol content as it did in steady state. This was not done by $S$. cerevisiae under either circumstance.

This work was supported by the award of a Research Associateship from the University of Wollongong.

\section{REFERENCES}

ANAND, J. C. (1969). The physiology and biochemistry of sugar-tolerant yeasts. Ph.D. thesis, University of New South Wales, Australia.

ANAND, J. C. \& Brown, A. D. (1968). Growth rate patterns of the so-called osmophilic and nonosmophilic yeasts in solutions of polyethylene glycol. Journal of General Microbiology 52, 205212.

AtzPodien, W. \& Bode, H. (1970). Purification and regulatory properties of ATP-sensitive phosphofructokinase from yeast. European Journal of Biochemistry 12, 126-132.
Batke, J., Asboth, G., Lakatos, S., Schmitt, B. \& COHEN, R. (1980). Substrate induced dissociation of glycerol-3-phosphate dehydrogenase and its complex formation with fructose-bisphosphate aldolase. European Journal of Biochemistry 107, 389-394.

Bewley, G. C. \& Miller, S. G. (1979). Origin and differentiation of the soluble $\alpha$-glycerolphosphate dehydrogenase isozymes in Drosophila melanogaster. In Isozymes: Current Topics in Biological and Medical Research 3, pp. 23-52. Edited by M. C. Rattazi, J. G. Scandalio \& G. S. Whitt. New York: Alan R. Liss Inc. 
BLACK, W. J. (1966). Kinetic studies on the mechanism of cytoplasmic $\mathrm{L}-\alpha$-glycerophosphate dehydrogenase of rabbit skeletal muscle. Canadian Journal of Biochemistry 44, 1301-1317.

Brown, A. D. (1978). Compatible solutes and extreme water stress in eukaryotic micro-organisms. $A d$ vances in Microbial Physiology 17, 181-242.

Brown, A. D. \& EdGLEY, M. (1980). Osmoregulation in yeast. In Genetic Engineering of Osmoregulation, pp. 75-90. Edited by D. W. Rains, R. C. Valentine \& A. Hollaender. New York: Plenum Press.

Davies, R. \& Wayman, F. (1973). Estimation of glucose and invertase activity in presence of thiols. Analytical Biochemistry 55, 143-153.

EDGLEY, M. (1980). Osmoregulation in xerotolerant and non-tolerant yeasts. Ph.D. thesis, University of Wollongong, Australia.

Edgley, M. \& Brown, A. D. (1978). Response of xerotolerant and non-tolerant yeasts to water stress. Journal of General Microbiology 104, 343-345.

Eggstein, M. \& KunlmanN, E. (1974). Triglycerides and glycerol. Determination after alkaline hydrolysis. In Methods of Enzymatic Analysis 4, pp. 18251831. Edited by H. U. Bergmeyer. New York: Academic Press.

Gancedo, C., Gancedo, J. M. \& Sols, A. (1968). Glycerol metabolism in yeasts - pathways of utilisation and production. European Journal of Biochemistry 5, 165-172.

Hanahan, D. J. \& Olley, J. N. (1958). Chemical nature of monophosphoinositides. Journal of Biological Chemistry 231, 813-828.

HofmanN, E. (1976). The significance of phosphofructokinase to the regulation of carbohydrate metabolism. Review of Physiological and Biochemical Pharmacology 75, 1-68.

LAYNE, E. (1957). Proteins and derivatives. III. Biuret method. Methods in Enzymology 3, 447-454.

NADER, W., BetZ, A. \& Becker J.-U. (1979). Partial purification, substrate specificity and regulation of $\alpha$-L-glycerolphosphate dehydrogenase from Saccharomyces carlsbergenesis. Biochimica et biophysica acta 571, 177-185.

Sols, A. \& Salas, M. L. (1970). Phosphofructokinase. III. Yeast. Methods in Enzymology 9, 436-442.

Storey, J. M. \& Storey, K. B. (1982). Kinetic properties and regulation of glycerol-3-phosphate dehydrogenase from the overwintering, freezingtolerant gall fly larva, Eurosta solidaginis. Cryobio$\log y$ 19, 185-194.

Tajima, K. \& Yoshizumi, H. (1973). Mechanism of glycerol and 2,3-butanediol formation by yeast in highly salted media during alcoholic fermentation. Journal of Fermentation Technology 51, 560-565. 